\title{
Soziales Elend nach Stopp oder Verweigerung von IV-Renten
}

\section{Doris Brühlmeier-Rosenthal}

Dr. med., Fachärztin für Psychiatrie und Psychotherapie, Mitglied FMH

Als praktizierende Psychiaterin gelangte die Autorin in den letzten Jahren zur Gewissheit, dass die systematische «härtere Gangart» der IV bei psychisch-psychosomatisch Kranken und Schmerzpatienten unter medizinischen und ökonomischen Gesichtspunkten kontraproduktiv ist. Eine von ihr durchgeführte «Nachzählung» erhärtete ihren Verdacht. Die erhobenen Zahlen liefern Indizien, mit denen sie eine vertiefte Diskussion des Problems anzuregen hofft.

\section{Scheininvalide?}

Es muss ca. 2005 gewesen sein: Die damals 59-jährige, schwer psychisch kranke Frau Z. sass zitternd und untröstlich weinend da: «Sie wollen uns die IV-Rente wegnehmen!» «Sicher, ganz sicher nicht», versuchte ich zu beruhigen, «wissen Sie, die Renten sind unantastbar.» «Doch doch, die grösste Partei will unsere Renten stoppen, es stand im (Tagi), wir heissen jetzt Scheininvalide.» "Niemals wird das möglich werden, in ganz Europa nicht. Wie gesagt: Die Renten sind und bleiben unantastbar.» Ich war überzeugt, das richtige Argument gefunden zu haben. Aber, wie recht die Patientin behalten sollte!

\section{Erste IV-Ausmusterung 2009}

Nur 4 Jahre später geschah es zum ersten Mal: Eine ebenfalls schwer psychisch und schmerzkranke Pa-

\section{Résumé}

En tant que praticienne en psychiatrie, l'auteure a, ces dernières années, acquis la certitude que le durcissement systématique par I'Al de sa position à l'égard des personnes atteintes de maladies psychiques/psychosomatiques et des patients douloureux est contre-productif sur les plans médical et économique. Un recomptage effectué par ses soins a renforcé ses soupçons. Les chiffres recueillis fournissent des indices avec lesquels elle espère lancer un débat approfondi sur le sujet. tientin, Frau D., wurde zu einem polydisziplinären Gutachten aufgeboten. Unverständlicherweise nur $6 \mathrm{Mo}$ nate nach der letzten erfolgreichen Rentenrevision, nach 14 Jahren IV-Rente. Nur 4 Monate später war die IV-Rente weg auf Ende des kommenden Monats. Die Patientin sei in angepasster Tätigkeit zu 100\% arbeitsfähig. Diese Einschätzung stand im Gegensatz zu 7 früheren psychiatrischen Gutachten und zu 13 Arztberichten. Wie sich später herausstellen sollte, als ich endlich begonnen hatte, die IV-Akten zu bestellen und trotz grossem Zeitaufwand durchzuarbeiten, war sie bei der IV denunziert worden, von einem 20 Jahre älteren Freund, der sie hatte heiraten wollen, wozu sie nicht in der Lage war. Die IV vertraute offenbar dieser Denunziation.

Es folgten ein psychiatrischer Klinikaufenhalt für $2 \mathrm{Mo}$ nate, wöchentlich Psycho-Spitex, $600 \mathrm{mg}$ Quetiapin pro Tag, eine Schulter-OP, kürzlich ein Fibula-Bruch und gehäufte psychiatrische Konsultationen. Ein Rekurs gegen den Entscheid wurde abgelehnt. Nach der IV-Ausmusterung stiegen die Krankheitskosten ungefähr um den Faktor 20. Der schlechte Zustand der Patientin hält seit 6 Jahren an, eine Gesundung oder die Wiedererlangung der Arbeitsfähigkeit sind nicht in Sicht ...

\section{Weitere Gesunderklärungen dank der Zahlen 38\%, 39\%, 70\%}

In der Folge kamen weitere Patienten mit gestrichenen oder reduzierten IV-Renten in meine Praxis. 2010 die 50-jährige Frau P. Seit 2002 hatte sie eine volle IV-Rente bezogen. 2010 wurde diese auf 50\% reduziert: Das 
dritte und vierte Psychiatrische Gutachten, beide vom gleichen Psychiater erstellt, hatten sie zu 50\% gesund erklärt, entgegen der Beurteilung durch 25 frühere Arztberichte und zwei vorhergehende Gutachten. Es folgten: «sozialer Tod», grosse Armut, schwere Depression, Rezidiv der Anorexie etc.

Fünf der sechs Patienten in meiner Praxis, deren IVRenten gekürzt wurden, sind alleinstehende Schweizerinnen im Alter von 53 bis 60 Jahren!

\section{IV-Rentner arbeiten zu einem relativ hohen Prozentsatz.}

Ich begann, die IV-Akten betroffener Patienten zu bestellen: Die Empörung wuchs mit jeder Akte: Gesunderklärungsgutachten bewerten in diesen Fällen die Arbeitsfähigkeit plötzlich mit $70-80 \%$ in angepasster Tätigkeit, d.h. keine Teilrente, keine Integrationsmassnahmen. Diese gibt es erst ab 40\% Arbeitsunfähigkeit. Die IV errechnete bei den Betroffenen aus 70\% Arbeitsfähigkeit in angepasster Tätigkeit (laut Gutachten) maximal 38 bis 39\% Arbeitsunfähigkeit.

Ein pikantes Beispiel: Ein von der IV wegen einer Arbeitsunfähigkeit von "nur» 39\% zurückgewiesener MS-Patient trifft in seiner MS-Selbsthilfegruppe zwei weitere MS-Kranke. Der eine ist auf den Rollstuhl angewiesen, der andere weist zusätzlich eine Hemiparese auf. Den beiden wurde von Gutachtern desselben Zentrums eine Arbeitsunfähigkeit von 38\% respektive $34 \%$ attestiert und sie wurden von der IV abgewiesen.

\section{Radikalisierung}

Frau H, eine schwer depressive und an Fibromyalgie leidende Patienten, die zudem durch wiederholte Inzest-Ereignisse traumatisiert war (u.a. wurde die Tochter vom Ehemann der Patientin geschwängert), bezog seit 2003 eine IV-Rente. Im November 2015 erhielt sie den folgenden IV-Bescheid: «Die Rente wird nach Zustellung der Verfügung auf Ende des folgenden Monats aufgehoben.» Mein Schreiben an die IV und entsprechende Gutachter wurde zurückgewiesen. Die Akte sei geschlossen ... Frau H. sei wahrscheinlich rein aus psychosozialen Gründen psychiatrisch hospitalisiert gewesen, stand im Bescheid. In angepasster Tätigkeit wäre sie zu 100\% arbeitsfähig, stand im Gesunderklärungsgutachten einer weiteren dafür bekannten MEDAS-Stelle.

Wut, Ohnmacht, erlebte Unfähigkeit und weitere IVDramen als Folge der Scheininvalidenkampagne miterleben zu müssen, veranlassten mich dazu, selbst aktiv zu werden und zu recherchieren, ob Berufskollegen in ihren Praxen Ähnliches erlebten.

\section{Nachzählung: Fakten statt Worte}

Die Schlagzeile: Abnahme der IV-Rentenzahl von 197000 im Jahr 2010 auf 177500 im Jahr 2015 (bei gleichzeitigem Bevölkerungswachstum von 0,5 Mio.) schmerzte. Erstmals in meinem schon länger dauernden Leben schrieb ich unter dem Titel «So viel Ungerechtigkeit gegenüber IV-Bezügern» einen Leserbrief an die Aargauer Zeitung, der am 11.6.2016 erschien. Natürlich änderte sich nichts.

Die Idee einer Nachzählung drängte sich mir auf, nach dem Motto: Mit Worten ist nichts zu erreichen, wie sehen denn eigentlich die Zahlen aus? Seit 28 Jahren bin ich in der psychiatrischen Praxis, viele meiner $\mathrm{Pa}$ tienten mit Voll- oder Teil-IV arbeiten. Wie viele sind es genau? Wir brauchen Zahlen. Ich sehe viele meiner früheren und jetzigen Patienten vor mir, die mit IVTeilrente bis zu ihrer Pensionierung Teilzeit gearbeitet haben und jene, die trotz voller IV ein paar Stunden pro Woche im ersten Arbeitsmarkt arbeiten.

Ich entwarf also einen Fragebogen und zählte nach. Bereits nach ca. 2 Wochen standen meine Praxiszahlen in auch für mich überraschender Klarheit da: IV-Rentner arbeiten zu einem relativ hohen Prozentsatz. In meiner Praxis waren 46\% der Vollrentner und 87\% der Teilrentner arbeitstätig. So gesehen ist die IV-Rente für meine Patienten ein Segen. Denn bei den ausgemusterten und von der IV zurückgewiesenen Patienten sieht das Bild anders aus. Diese sind zu $52-100 \%$ vom Sozialamt abhängig und maximal zu 5\% arbeitsfähig. Aus meiner Sicht ist die Rentenverweigerung eindeutig als Fluch zu sehen, der nicht zu Arbeitsfähigkeit führt! Nach diesen eindeutigen Befunden für meine Praxis stellte sich die Frage, ob es in anderen Praxen ähnlich wäre. Nachfragen bei einem "Armutsforscher», bei

Ich will nicht weiterhin von Patienten hören müssen: «Die IV will, dass es mich nicht mehr gibt. Warum sagen sie uns nicht direkt «Bringt euch doch um!?»"

psychiatrischen Spezialisten und Recherchen im Internet bestätigten meine Vermutung. Es gab bis anhin keine Nachuntersuchung psychiatrischer, neurologischer und von Schmerzpatienten, denen die IV verweigert wurde. Dabei ist diese Zahl mit mindestens 20000 Patienten in den vergangenen 4 Jahren beträchtlich.

\section{Kohärente Zahlen}

Ende September 2016 verschickte ich schliesslich 50 Fragebogen an praktizierende Psychiater aus den Kantonen Aargau und Zürich. Nach 2 Wochen kamen die 


\section{Einige Zahlen und Fakten zur IV}

- 4.-6. IV-Revision: Die Zahl der IV-Rentner muss sinken, 17500 Rentner sollen in den 1. Arbeitsmarkt integriert werden.

- Die Zahl der IV-Neurentner hat sich seit 2003 um die Hälfte reduziert auf ca. 14000 pro Jahr. Die Bevölkerungszahl der Schweiz stieg seit 2010 um 455000 auf 8,32 Mio. (In jeder Population gibt es 1-1,5\% Schizophreniekranke, d.h. seit 2010 ca. 6000 mehr.)

- 1628 CHF beträgt die durchschnittlich IV-Rente pro Monat.

- Wer die IV verliert, verliert auch den geschützten Arbeitsplatz, die eventuelle 2. Säule, das Recht auf Ergänzungsleistungen.

- Die Gemeinden Schlieren und Dietikon beauftragen Juristen, um doch noch eine Rente für ihre kranken Einwohner zu erreichen.

ersten ausgefüllten Bogen zurück, aus dem Aargau, aus dem Zürcher Säuliamt und aus der Stadt Zürich.

Die Resultate waren bei allen sehr ähnlich wie in meiner Praxis. Offensichtlich ist hier eine humanitäre Katastrophe im Gange. Im November begann ich zusammenzuzählen, Graphiken zu entwerfen, nächtelang zu schreiben. Insgesamt flossen 13 Fragbögen mit 402 Patienten in die Auswertung ein (Rücklaufquote $24 \%)$.

\section{Rente als Segen, Rentenverweigerung als Fluch}

Auch wenn es sich bei meiner Umfrage nicht um eine wissenschaftliche Studie handelt, haben die Resultate von 402 Patienten, davon 177 «IV-Opfer», zweifellos eine gewisse Aussagekraft. Es zeigt sich klar, dass IV-Ausmusterung (43 Patienten) in 93\% direkt in den «sozialen Tod» führt, mit Sozialamtabhängigkeit, vermehrter Krankheit, Hospitalisationen, Armut, vollkommener Erwerbsunfähigkeit. Rentenverweigerung

1 In der Online-Ausgabe sind die erhobenen Zahlen zusätzlich graphisch in Form von Kuchendiagrammen visualisiert.

Korrespondenz:

Dr. med. Doris Brühlmeier-

Rosenthal

Fachärztin für Psyhiatrie

und Psychotherapie

Uitikonerstrasse 4

CH-8952 Schlieren

doris.bruehlmeier[at]

gmail.com
(134 Patienten) führte in 60\% zum sozialen Tod oder ähnlich negativen Entwicklungen: Nur 4\% der Betroffenen arbeiten im geschützten Rahmen. Dass Rentenverweigerung zu Erwerbstätigkeit führt, ist in meiner Umfrage 177-fach widerlegt.

Rentenzusprache (225 Patienten) ist bei psychiatrischen, neurologischen und Schmerzpatienten ein Segen: 36-48\% der IV-Rentner arbeiten Teilzeit, zumindest die Kosten für psychiatrische Betreuung betragen nach Berentung nur noch ca. $1 / 10$ der Kosten vor Berentung. Die Krankheit stabilisiert sich, es kommt zu weniger Rezidiven, weniger Psychopharmaka sind nötig.

\section{Forderungen}

Jeder Tag, an dem weitere Patienten IV-Opfer werden, ist ein verlorener Tag. Ich will nicht weiterhin von $\mathrm{Pa}$ tienten hören müssen: «Die IV will, dass es mich nicht mehr gibt. Warum sagen sie uns nicht direkt «Bringt euch doch um!??»

Deshalb meine Forderungen:

- Sofortiger Stopp und Änderung des jetzigen IV-Verweigerungsverfahrens. Es gibt weder einen therapeutischen, noch einen sozialen oder wirtschaftlichen Wert der Rentenverweigerung, was durch meine Nachzählung 177-fach untermauert wird.

- Sofortige Wiedergutmachungen, das heisst Weiterführen der IV-Rente bei ungerecht Ausgemusterten. Einrichtung eines Wiedergutmachungs-Fonds, bis die IV-Renten wieder zugesprochen werden.

- Renten statt Gesunderklärungsgutachten (81 Mio. Franken ergibt 4000 IV-Jahresrenten [1]).

- Zusammenschluss von uns Ärzten, Therapeuten und Juristen, um politisch die nötigen Veränderungen zu erreichen.

- Rentenentscheide durch zwei bis drei behandelnde Ärzte im Kollektiv fällen.

- Krankenkassenbeiträge steigen jährlich, IV-Beiträge sollen auch steigen dürfen von derzeit 1,4\% z.B. auf $1,7 \%$. Es darf keine weiteren IV-Opfer geben.

- Listen der IV-Opfer aus unseren Praxen erstellen und gemeinsam die IV zurückfordern.

\section{Epilog}

Mit meiner Nachzählung, habe ich, wie zu erwarten war, bisher noch nichts erreicht. Heute kommt die Nachricht einer Kollegin: Eine 43-jährige Patientin mit Mischpsychose, 3 Kindern und einem Ehemann wurde notfallmässig mit schwerem Rezidiv in die Klinik gewiesen. Ihre langjährige IV-Rente wurde vor 2 Monaten eingestellt. 2016 wurden übrigens nur schon im Kanton Aargau 526 IV-Renten gestrichen.

\section{Referenz}

1 Die Zahl von 81 Millionen Franken umfasst alle IV-Gutachten, nebst den "Gesunderklärungsgutachten» auch jene, die eine IV ermöglichten. Quellen: medinside.ch vom 26.01.2017, Prof. Regina Kunz / SRF News vom 25.10.2016: www.srf.ch/news/schweiz/ iv-rente-als-lotterie-grosse-unterschiede-je-nach-gutachter Da die durchschnittliche IV-Rente pro Jahr $12 \times 1628 \mathrm{CHF}=20536 \mathrm{CHF}$ beträgt, entsprechen die 81 Millionen Franken, die jährlich für Gutachten aufgewendet werden, ca. 4000 Jahresrenten. 